for several sleep disorders where single night polysomnography is insufficient (ICSD-2). However, the optimal recording time for measurement remains unclear. We hypothesised that 7 days would provide similar data as recording for 14 days.

Patients \& Methods We analysed 3 consecutive years of actigraphy data obtained within a tertiary sleep referral centre. Data were recorded continuously for two weeks using the AW2 Actiwatch (Cambridge NeuroTechnology, UK), using Actiwatch Sleep Analysis 5 software for analysis of the data. Parameters, including sleep efficiency (SE), sleep latency (SL), sleep fragmentation index (SFI), total sleep time (TST) and wake after sleep onset (WASO), were classified into week 1 , week 2 and an overall average for the duration of 14 days. In addition, two experienced consultants working in the sleep laboratory compared the results of week 1 vs. week 2 independently, visually analysing the data for circadian rhythmicity and fragmentation of the pattern; allowing calculation of the itraclass correlation coefficient (ICC), $\kappa$.

Results The actigraphies of 239 patients (51.9\% (124) male; mean age 42 (16) years) were analysed. There was no difference in SE, SL, SFI or WASO between week 1 , week 2 and 14 days average recording. A difference was found between TST week 1 (399.9, 95\% CI 389.9-409.9 mins) and TST week 2 (388.7, 95\% CI 378.3-399.1 mins), but not between TST for 14 days average recording and either week (394.3, 95\% CI 384.7-403.9 mins). Independent scorers achieved a strong agreement in the rhythmicity of the sleep pattern (ICC $\kappa 0.734, p<0.001$ ) and a low agreement for the fragmentation of the pattern (ICC $\kappa 0.380, \mathrm{p}<0.001$ ).

Conclusion One week of actigraphy provides similar data to two week actigraphy, despite subtle differences between different weeks. One week actigraphy should be recommended as standard to maximise efficiency of the clinical service.

\section{S45 SLEEP DISORDERED BREATHING (SDB) IN PATIENTS OF TYPE 2 DIABETES MELLITUS(DM) WITH AND WITHOUT RETINOPATHY- A HOSPITAL BASED STUDY}

doi:10.1136/thoraxjnl-2012-202678.051

S Mehta, D Chaudhry, SV Singh, A Atreja, V Sangwan. Pt BD Sharma PGIMS, Rohtak, India

Background Higher incidence of SDB has been reported from West in patients having sight threatening retinopathy with limited information from South Asia.

Methods It was a prospective, observational study carried out on 80 patients of type $2 \mathrm{DM}$ presented to Vitreo-retinal clinic. Patients with coronary artery disease, acute and chronic Heart Failure, concurrent hypothyroidism, known Obstructive sleep apnea (OSA) with DM and of any known respiratory disorder were excluded.

On the basis of fundus and fluorescein angiography they were divided into two Groups, Group I without retinopathy $(n=30)$ and Group II with retinopathy $(n=50)$. Latter had 30 and 20 patients of Non Proliferative Diabetic retinopathy (NPDR) and Proliferative Diabetic retinopathy (PDR) respectively. All patients underwent whole night attended polysomnography in the hospital.

Results The patients having diabetic retinopathy had higher age, BMI, waist circumference, Serum creatinine, HbA1c and Apnea Hypoapnea index (AHI) $(p<0.05)$. PDR sub-group had a higher waist and neck circumference however NPDR patients had a higher HbA1c $(p<0.05)$. OSA was observed in $66 \%$ and $40 \%$ of patients with \& without diabetic retinopathy $(12 / 30$ vs. $33 / 50)$ respectively. On subgroup analysis, $90 \%$ of patients having PDR had OSA as compared to $50 \%$ in NPDR group. Severity of OSA was proportional to the presence of retinopathy (AHI $=24.35 \pm 27.72$ vs. $13.09 \pm 20.3$ in Gp II \& Gp.I respectively). It was nearly twice as severe in PDR Group than NPDR Group
((34.91 \pm 30.61 vs $17.3 \pm 23.94)$ with significantly higher desaturation $(5.37 \pm 5.09 \%$ vs. $11.53 \pm 6.82 \%)$. On multivariate regression analysis only $\mathrm{HbA1c}$ levels correlated with presence of SDB in Diabetics.

Conclusion In a selective hospital based population of type $2 \mathrm{DM}$, prevalence and severity of SDB correlated with severity of retinopathy. A larger longitudinal study is need of the hour to predict factors responsible for OSA and find correlation between SDB with diabetic retinopathy.

Key words sleep disordered breathing, type 2 Diabetes Mellitus, NPDR, PDR, Apnea Hypoapnea Index.

\section{S46 THE RISK OF OBSTRUCTIVE SLEEP APNOEA IS SIGNIFICANTLY HIGHER IN PATIENTS WITH CHRONIC KIDNEY DISEASE - A STUDY FROM A SINGLE UK RENAL CENTRE}

doi:10.1136/thoraxjnl-2012-202678.052

AW Seccombe, P Ferguson, J Baharani. Heartlands Hospital, Birmingham, UK

Introduction and Objectives Along with known associations with coronary heart disease, heart failure and stroke, there is increasing evidence to demonstrate a link between obstructive sleep apnoea (OSA) and chronic kidney disease (CKD). There is also growing evidence to suggest that intensive treatment of CKD can improve or even eliminate symptoms related to OSA. So far though, there is no evidence to support the association between OSA and CKD in a UK population. Our study aimed to quantify the risk of OSA in different severities of CKD for the first time in the UK Our secondary aim was to look for factors that increased or decreased this risk, including medications.

Methods Using the renal database, 60 patients each at CKD stage $3 b, 4$ and 5, haemodialysis patients and renal transplant patients were identified, matched for age and sex and sent the Berlin questionnaire and a lifestyle questionnaire. The Berlin questionnaire was used to stratify patients into high-risk and low-risk groups for OSA. We used the lifestyle questionnaire along with up-to-date laboratory investigations to investigate variables that altered this risk within our sample.

Results Of the 300 patients who were contacted, 111 (37\%) responded. Of the 111 respondents, $78(70.2 \%)$ were found to be at high-risk of OSA. This significant increase in risk was seen in all severities of CKD that were examined including in CKD $3 \mathrm{~b}$ (80.8\%). In contrast to the general population, where OSA is more common in men, our study found that $77.1 \%$ of women were at high-risk of OSA compared to $65.1 \%$ of men. We also found that renin-angiotensin system inhibitors (RASI) were associated with a reduced risk of OSA with $66.1 \%$ of users being at high risk compared to $74.5 \%$ of non-users.

Conclusions Our study is the first to demonstrate the link between OSA and CKD in a UK population, a link that holds for milder forms of CKD. We noted that RASI use helped to reduce the risk of OSA, thus identifying a possible medical treatment for OSA in patients with CKD. Further study will clarify the importance of this treatment.

\section{S47 COMPARING COPING STRATEGIES WHILE DRIVING IN PATIENTS WITH OBSTRUCTIVE SLEEP APNOEA SYNDROME (OSAS) AND IN HEALTHY CONTROLS}

doi:10.1136/thoraxjnl-2012-202678.053

'A Dwarakanath, 'D Ghosh, ${ }^{2} \mathrm{SL}$ Jamson, 'MW Elliott. 'St James' University Hospital, Leeds, UK; 'Institute for Transport Studies, University of Leeds, Leeds, UK

Introduction Tiredness while driving is potentially fatal and it is recommended that a driver who starts to feel tired should stop and 